Erratum

\title{
Erratum to "miR-876 Inhibits EMT and Liver Fibrosis via POSTN to Suppress Metastasis in Hepatocellular Carcinoma"
}

\author{
Kai Chen, ${ }^{1,2}$ Zhonghu Li, ${ }^{3}$ Mengyun Zhang, ${ }^{4}$ Bo Wang, ${ }^{3}$ Tao Peng, ${ }^{5}$ Yanbing Shen, ${ }^{3}$ \\ Jianxin Zhang, ${ }^{3}$ Jiaxin Ye, ${ }^{3}$ Yu Liu, ${ }^{2}$ Di Tang, ${ }^{3}$ Minjie Peng, ${ }^{6}$ Dandan Ma, ${ }^{3}$ Zhengkang Xiao, ${ }^{3}$ \\ Yujun Zhang, ${ }^{1}$ Weidong Jin, ${ }^{3}$ and Xiaowu Li $\mathbb{C}^{1,6}$ \\ ${ }^{1}$ Hepatobiliary Surgery Institute, Southwest Hospital, Army Medical University, China \\ ${ }^{2}$ Department Hepatobiliary Surgery Institute, Chengdu Fifth People's Hospital, China \\ ${ }^{3}$ Department General Surgery, Central Theater Command General Hospital of PLA, China \\ ${ }^{4}$ Department Rheumatology of Integrated Traditional Chinese and Western Medicine, \\ Central Theater Command General Hospital of PLA, China \\ ${ }^{5}$ Department of Hepatobiliary Surgery, The First Affiliated Hospital of Yangtze University, Jingzhou, China \\ ${ }^{6}$ Hepatobiliary Surgery \& Carson International Cancer Shenzhen University General Hospital \& Shenzhen University Clinical \\ Medical Academy Center, Shenzhen University, China \\ Correspondence should be addressed to Xiaowu Li; lixw1966@163.com
}

Received 2 December 2020; Accepted 2 December 2020; Published 12 December 2020

Copyright ( 2020 Kai Chen et al. This is an open access article distributed under the Creative Commons Attribution License, which permits unrestricted use, distribution, and reproduction in any medium, provided the original work is properly cited.

In the article titled "miR-876 Inhibits EMT and Liver Fibrosis via POSTN to Suppress Metastasis in Hepatocellular Carcinoma" [1], information was omitted from the Acknowledgments section. The corrected Acknowledgments section is shown below. This mistake was introduced during the production of the article, and the publisher apologizes for this error.

\section{Acknowledgments}

The majority of the work was supported by the National Natural Science Foundation of China under Grant 81430063 and the National Natural Science Foundation for Young Scientists of China under Grant 81902501.

\section{References}

[1] K. Chen, Z. Li, M. Zhang et al., "miR-876 inhibits EMT and liver fibrosis via POSTN to suppress metastasis in hepatocellular carcinoma," BioMed Research International, vol. 2020, Article ID 1964219, 16 pages, 2020. 